\title{
V-I CONVERTERS WITH TRANSCONDUCTANCE PROPORTIONAL TO BIAS CURRENT IN ANY TECHNOLOGY
}

,

\author{
Vladimir I. Prodanov
}

Bell Laboratories/ Lucent T ec hnologies
600 Mountain Ave., Murray Hill, NJ 07974

\begin{abstract}
In this paper we discuss circuits comprised of multiple differential pairs driven in parallel. We show that high linearit $y$ and linear with bias current tunability are intrinsic properties of an y equidistant-offset multi-pair circuit. We argue that these tw o properties could poten tially allow us to implement designs of analog filters, multipliers and other circuits that are (in first-order) technology independent. Simulations results are provided in support of presented simple intuitiv e explanation.
\end{abstract}

\section{INTRODUCTION}

Architectures using parallel combination of differential pairs, where each pair has suitably chosen offset voltage, have been studied and used extensively (see [1] and its many references). Due to the fact that bipolar transistor and for that reason bipolar differential pair have well-defined $V-I$ characteristics ( $\tanh$ for the second one) most of the reported multi-pair structures are bipolar. Those, often called "multi-tanh" circuits [1], [2], ha ve been used for sythesis of trigonometric functions [3], [4] and design of amplifiers with gain inv ersely proportional to control signal [5]. Multi-tanh circuits have also be used to implement con tin uous-time filters [6] and analog multipliers [7]. The properties that make multi-tanh circuits useful in filtering and analog multiplication applications are their line arityand line ar tuning capability (transconductance proportional to bias current).

The aim of this paper is to show that linear (with respect to bias current) tuning is property of any equidistant offset multi-pair transconductor and not only of those implemented in bipolar technology. The importance of the above stated fact is that multi-pair based circuits can be migrated from one technology to another without significant change in their operation.

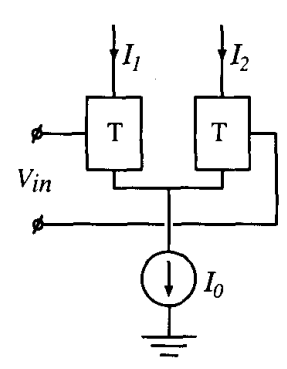

(a)

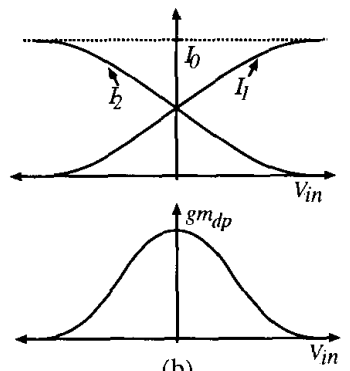

(b)
Figure 1: Differential Pair Circuit: (a) schematic; (b) voltage-current and transconductance characteristics;

\section{FUNDAMENTAL PROPERTIES OF SIMPLE DIFFERENTIAL $P$ AIRS}

In this section we will identify those diff.-pair properties that are fundamental for the operation of a multi-pair equidistant-offset circuit.

In Fig. 1(a) a differential-pair circuit is shown. The reason for depicting the used devices as "boxes" is to emphasized the fact that no assumptions about the used technology (bipolar or MOS) will be made. We will however assume that the tw odevices comprising the diff. pair are identical, i.e. perfectly matched. Under this condition the incremental transconductance of the diff.-pair circuit,

$$
g m_{d p}\left(V_{i n}\right)=\left|\frac{\partial I_{1}}{\partial V_{i n}}\right|=\left|\frac{\partial I_{2}}{\partial V_{i n}}\right|
$$

is symmetric function of $V_{i n}$. The shape of the $g m$ curve strongly depends on voltage-current characteristics of the used devices. The area under the curve, however; is completely independent of the device characteristics. This area equals the absolute change of $I_{1,2}$; which, as depicted in Fig. 1, is the tail current $I_{0}$. Thus,

$$
\int_{-\infty}^{\infty} g m n_{d p}(x) d x=I_{0}
$$




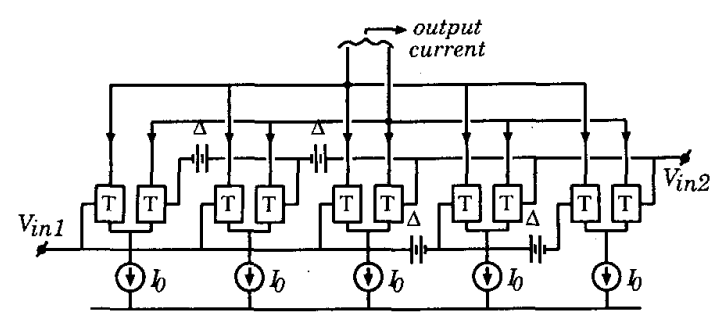

Figure 2: Conceptual Schematic of Multi-pair Equidistant-offset Circuit.

The symmetry of the $g m_{d p}$ characteristic and the constancy of the area under the $g m_{d p}$ curv eare the tw o properties we will base our further discussion upon.

\section{PROPERTIES OF EQUIDISTANT-OFFSET MULTI-PAIR ARCHITECTURES}

The conceptual schematic of a multi-pair structure having equidistant offsets is shown in Fig. 2. The structure consists of many identical differential pairs, each having w ell-defined offset vltage. The pair in the middle does not have an offset while all others do. As we move off the central pair the offset increases progressively taking values $\pm \Delta, \pm 2 \Delta$, etc.

T osimplify the discussion w ewill first consider a hypothetical multi-pair circuit consisting of infinitely many differential pairs.

\subsection{The infinite-pair case}

As sho wnin Fig. 3(a) the transconductance function of an infinite-pair circuit is the sum of infinitely many iden tical $\Delta$-spaced $g m_{d p}$ functions, i.e.

$$
g m_{m d p}\left(V_{i n}\right)=\sum_{k=-\infty}^{\infty} g m_{d p}\left(V_{i n}+k \Delta\right)
$$

The transconductance is obviously even periodic function of $V_{i n}$ (period of $\Delta$ ). Thus, the resulting transconductance function can be written in the form:

$$
g m_{m d p}\left(V_{i n}\right)=\sum_{k=0}^{\infty} a_{k} \cos \left(2 \pi k \frac{V_{i n}}{\Delta}\right)
$$

where all $a_{k}$ coefficients ha ve dimensions $A / V$.

The above expression can also be written as follows:

$$
g m_{m d p}\left(V_{i n}\right)=a_{0}\left[1+R\left(V_{i n}\right)\right]
$$

Here $R\left(V_{i n}\right)=\sum_{k=1}^{\infty} \frac{a_{k}}{a_{0}} \cos \left(2 \pi k \frac{V_{i n}}{\Delta}\right)$ is a dimensionless quantity that averages to zero in one $\Delta$ period. Function $R\left(V_{i n}\right)$ describes the variation of $g m_{m d p}$. For that

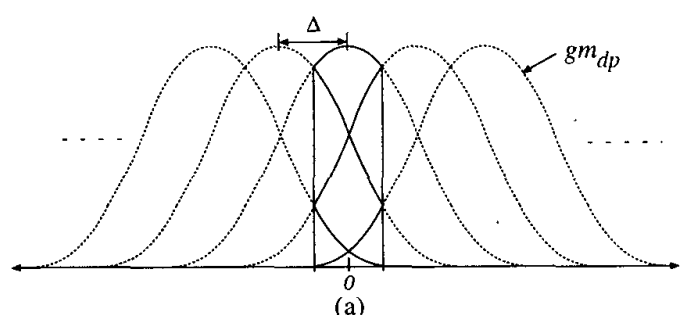

(a)

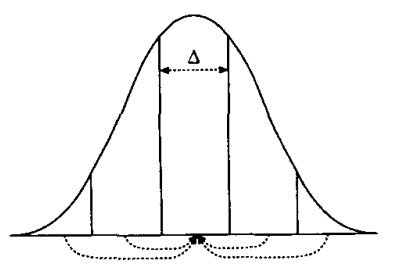

(b)

Figure 3: T ransconductance of a multi-pair circuit $\left(g m_{m d p}\right)$ having equidistant offset voltages : (a) $g m_{m d p}$ as a sum of iden tical $\Delta$-spaced $g m_{d p}$ functions. (b) viewing $g m_{m d p}$ in a $\Delta$ region as "sliced \& overlaid" $g m_{d p}$ function.

reason we will call it "the ripple" of $g m_{m d p}$. Because of the periodicity it suffice to consider the behavior of $g m_{m d p}$ only in the region $\pm \frac{\Delta}{2}$. Upon such consideration, we find the following: the $g m_{m d p}$ in theregion $\pm \frac{\Delta}{2}$ can be viewed (see Fig. $3(\mathrm{~b})$ ) as a result of slicing a single $g m_{d p}$ function into $\Delta$-pieces and ov erlaying them on top of each other. Thus, the area under the $g m_{m d p}$ curve in the region $\pm \frac{\Delta}{2}$ equals the total area under a single $g m_{d p}$ curve.

$$
\int_{-\frac{\Delta V}{2}}^{\frac{\Delta V}{2}} g m_{m d p}(x) d x=\int_{-\infty}^{\infty} g m_{d p}(x) d x=I_{0}
$$

Using equation (6) it is trivial to show that $a_{0}$ is given by:

$$
a_{0}=\frac{I_{0}}{\Delta}
$$

Thus, $a_{0}$ depends only on the biasing $\left(I_{0}\right.$ and $\left.\Delta\right)$ and not on the $V-I$ characteristic of the used transistors, i.e. the average (over one $\Delta$ period) transconductance value is independent of technology. The same result can be obtained in mathematically more rigorous $\mathrm{w}$ a $\frac{1}{y}$.It can also be shown that the higher-order coefficients do depend on $V-I$ characteristic of the used devices. Luckily, all those coefficients and as a result the ripple

\footnotetext{
${ }^{1}$ An excellent frame work for studying the relationship betw een $g m_{m d p}$ and $g m_{d p}$ results from the observation that $g m_{m d p}$ can be viewed as convolution of $g m_{d p}$ and an infinite tran of $\Delta$ spaced $\delta$ functions.
} 


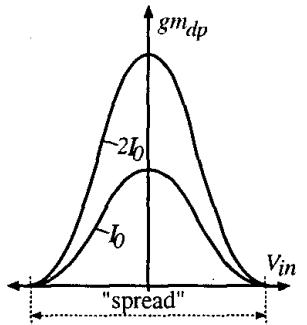

(a) (b)

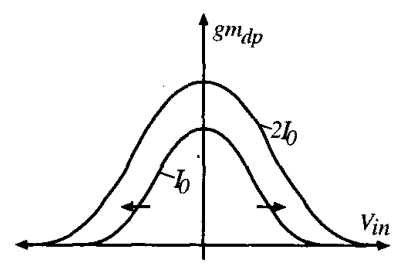

Figure 4: Qualitative depiction of the affect that tail current has on transconductance characteristic: (a) for BJT; (b) for MOS transistor;

$R\left(V_{i n}\right)$ are also functions of $\Delta$ and diminish rapidly with its reduction. Thus, high linearity (small $R\left(V_{i n}\right)$ ) can be achieved by proper selection of the offset voltage.

Assume that $\Delta$ is selected such that $R_{\max }=$ $\frac{\Delta}{I_{0}}\left|\sum_{k=1}^{\infty} a_{k}\right|$ is muc $\mathrm{h}$ smaller than 1 , then we can write:

$$
g m_{m d p}\left(V_{i n}\right) \approx a_{0}=\frac{I_{0}}{\Delta}
$$

This equation clearly sho ws that as a multi-pair circuit is being linearized its effective transconductance becomes:

1) linearly dependent on the bias current.

2) insensitive to the $V-I$ characteristics of the used activ e devices, i.e to tednology.

The above equation also suggests that the transconductance can be made nearly temperature, supply and process independent by making $I_{0}$ and $\Delta$ temperature, supply and process independent. This task can be accomplished in any tec hnology using standard band-gapbased bias techniques.

\subsection{The practical finite-pair case}

The transconductance of a multi-pair circuit employing finite number of differential pairs has three different regions: a middle region and tw oend regions. The middle region is the range of input voltages $V_{\text {in }}$ for which the following holds:

$$
\int_{V_{i n}-\frac{\Delta}{2}}^{V_{i n}+\frac{\Delta}{2}} g m_{m d p}(x) d x=I_{0}
$$

Hence, in this region the operation and the properties of the finite-pair circuit are identical to those of an infinite-pair one. Depending on the number of used pairs, selected technology and $\Delta$ such region may or may not exist.

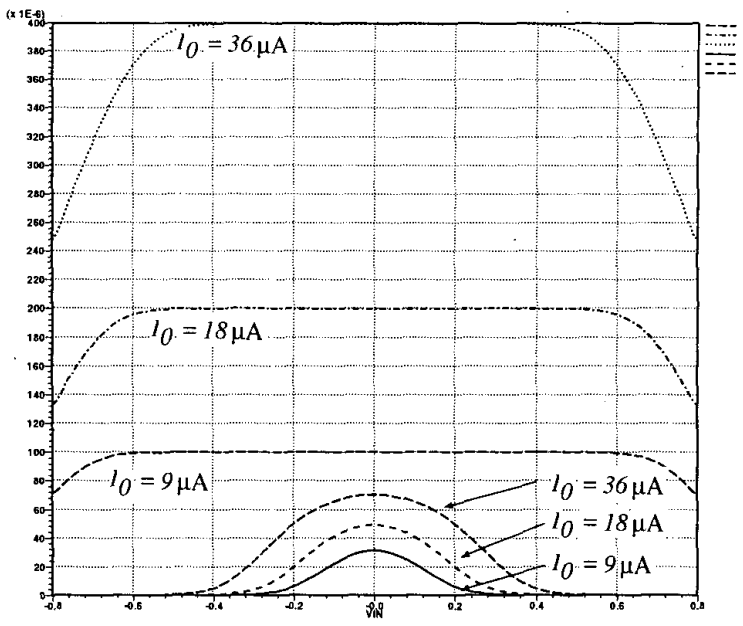

Figure 5: T ransconductance of 19-pair equidistantoffset MOS circuit $(\Delta=90 \mathrm{mV})$ and that of one of its diff. pairs for three different bias currents (simulation).

The affect of having finite number of pairs is observed in the "end regions". There

$$
\int_{V_{i n}-\frac{\Delta}{2}}^{V_{i n}+\frac{\Delta}{2}} g m_{m d p}(x) d V_{x}<I_{0}
$$

and $g m_{m d p}$ has $\mathrm{v}$ alue that is smaller than the nominal (and desired) $I_{0} / \Delta$. The end regions of a bipolar circuit do not change with $I_{0}$ while those of a MOS circuit grow with $I_{0}$. This difference is direct consequence of the fact that the spread of $g m_{d p}$ of a bipolar diff. pair does not change with $I_{0}$ (see Fig. 4 (a)) while the spread of the $g m_{d p}$ of a MOS diff. pair (as depicted in Fig. 4(b) and demonstrated in Fig. 5) increases with $I_{0}{ }^{2}$.

Fig. 5 simulation results are for $0.25 \mu \mathrm{m}$ CMOS process. Diff. pair transistors ha ve $L=0.32 \mu \mathrm{m}$ and $W=1 \mu \mathrm{m}$. Ideal voltage sources were used to implement desired $\Delta s$.

As shown in Fig. 5 the increase of the end regions in MOS multi-pair circuits causes decrease of the av ailable mid region. Nevertheless multi-pair MOS circuits can pro vide reasonable input linear range and a decade of linear-with-current tuning.

Fig. 6 compares the transconductances of tw o19pair CMOS circuits. Both circuits have the same $\Delta$ and $I_{0}$. The used diff. pair transistors how ev etha vedifferen $\mathrm{t}$ aspect ratios $(032 \mu \mathrm{m} / 1 \mu \mathrm{m}$ and $0.32 \mu \mathrm{m} / 2 \mu \mathrm{m})$. Fig. 6 plots clearly demonstrate the fact that mid-

\footnotetext{
${ }^{2}$ in square-root fashion in strong inversion and linearly in the regime of mobility saturation.
} 


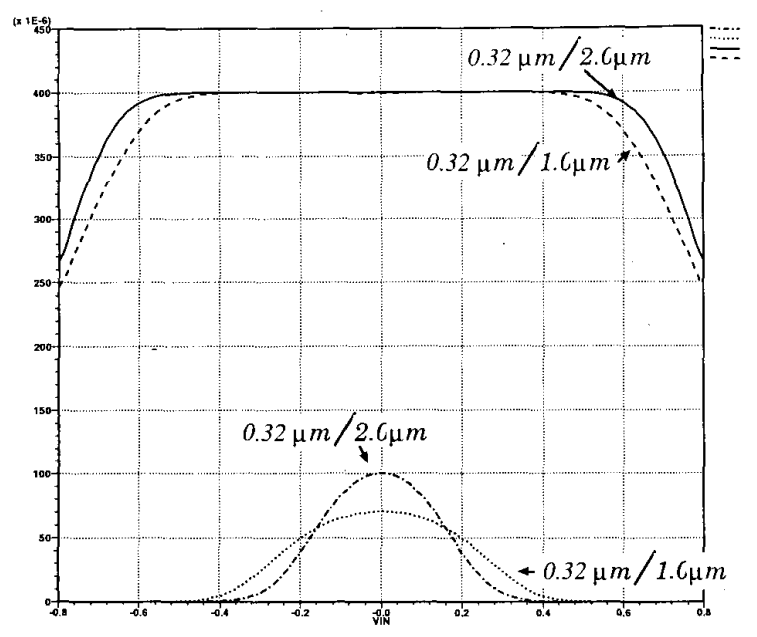

Figure 6: Transconductance of 19-pair MOS circuit and that of one of its diff. pairs for tw odifferent device widths (simulation).

region transconductance is mainly determined by biasing (e.g. $\Delta$ and $I_{0}$ ) and it is little sensitive to $V-I$ characteristics ofised devices. This suggests that no matter what technology is used Fig. 7 bias arrangement will deliver predictable and nearly constant (over temperature and process variation) transconductance. The last was verified (via simulation) during the design of a decade-tunable $(5-50 \mathrm{MHz}) \mathrm{Gm} / O T \mathrm{~A} / \mathrm{C}$ CMOS filter. Experimental results are currently being collected.

\section{CONCLUSIONS}

We have shown that linearity and linear (with bias current) tuning are properties of any multi-pair equidistantoffset circuit and not only to those implemented in bipolar technologies. This property can potentially allow us to implement designs of analog filters, multipliers and other circuits that are (in first-order) technology independent.

\section{REFERENCES}

[1] B. Gilbert,"The multi-tanh principle; a tutorial overview," IEEE Journal of Solid-State Circuits, vol. 33, pp. 2-17, Jan. 1998.

[2] B. Andersen, "The 'multi-tanh' technique for linearizing the transconductance of emitter coupled pairs," M. Sc. thesis, Washington State University , 1978.

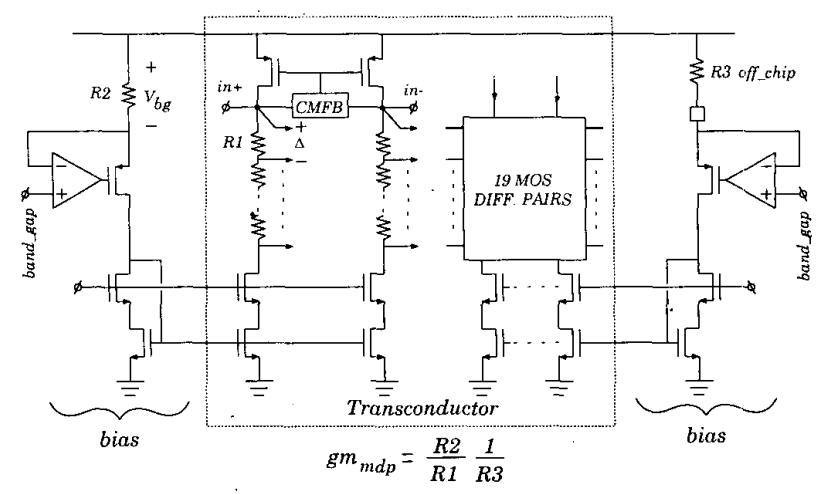

Figure 7: Conceptual schematic of bias arrangement of multi-pair transconductor used $\mathrm{Gm} / O T \mathrm{~A} / \mathrm{C}$ filter.

[3] B. Gilbert, "Circuits for the precise synthesis of the sine function," Electronic L etters, vol. 13, No. 17, pp. 506-508, Aug. 1977.

[4] B. Gilbert,"A monolithic microsystem for analog synthesis of trigonometric functions and their inverses;" IEEE Journal of Solid-State Circuits, vol. 17, pp.1179-1191, Dec. 1982.

[5] P. van Lieshout and R. van de Plassche. "A monolithic wideband variable-gain amplifier with a high gain range and low distortion," ISSCC Technical Digest, 1996, pp. 358-359.

[6] H. Tanimoto et al.,"Realization of a 1-V active filter using a linearization technique employing plurality of emitter-coupled pairs," IEEE Journal of SolidState Circuits, vol. 26, pp. 937-945, July 1991.

[7] K. Lasanen, J. T ervaluoto,A. Ruha and J. Roning, "A structure for extending the linear input voltage range of a differential input stage," $P r$ c. International Conference of Electronics, Circuits and Systems, 1998, vol. 2 pp. 355-358. 